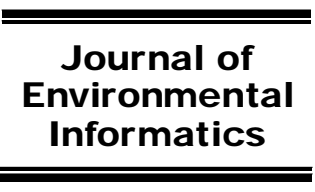

www.iseis.org/jei

\title{
A Robust Two-Step Method for Solving Interval Linear Programming Problems within an Environmental Management Context
}

\author{
Y. R. Fan and G. H. Huang ${ }^{*}$ \\ Institute for Energy, Environment and Sustainability Research, UR-NCEPU, North China Electric Power University, Beijing 102206, China
}

Received 9 October 2011; revised 25 December 2011; accepted 28 Febrary 2012; published online 12 March 2012

\begin{abstract}
In this study, a robust two-step method (RTSM) is developed to solve the interval linear programming (ILP) problem. It improved upon the two-step method (TSM) proposed by Huang et al. (1992) through incorporating additional constraints into solution procedures to avoid absolute violation. RTSM was applied to a simple case related to environmental management. The results demonstrated its applicability of the developed methodology. Compare with the modified interval linear programming (MILP) method proposed by Zhou et al., (2008) and the three-step method (ThSM) developed by Cao and Huang (2011), RTSM can generate a relatively larger solution space and thus avoid significant loss of decision-related information. Besides, RTSM has simpler solution procedures than ThSM, and will not lead to great computational requirement.
\end{abstract}

Keywords: decision support, algorithm, interval number, optimization, uncertainty

\section{Introduction}

Optimization is a useful tool for supporting effective environmental management (van Beek et al., 1992; Bloemhof-Ruwaard, et al., 1995; Huang and Chang 2003). However, in many real-world environmental management problems, uncertainties exist in various system components and their interrelationships. For example, waste generation rate within a city is related to many socio-economic and environmental factors, and exhibits uncertain and dynamic features; the efficiency of a municipal wastewater treatment plant is affected by wastewater flow rate, and is uncertain in nature; regional air quality is mainly influenced by air pollutant emissions within this area, which also present uncertain characteristics. Such uncertainties can lead to increased complexities in the related optimization efforts. Simply ignoring these uncertainties is considered undesired as it may result in inferior or wrong decisions (Ruszcyński, 1997; Young, 2001; Ozdemir and Saaty, 2006; Zou, et al., 2010). Therefore, inexact optimization methods are desired for supporting environmental management under uncertainty.

In the past decades, amounts of optimization methods were developed for environmental management under uncertainty, mainly including stochastic, fuzzy and interval mathematical programming (abbreviated as FMP, SMP and IMP, respectively) (Chang and Wang, 1997; Huang et al., 1992; Chang and Lu, 1997; Karmakar and Mujumdar, 2006a, b; Li et al., 2006,

${ }^{*}$ Corresponding author. Tel.: +86 10 61772018; fax: +86 1051971284 .

E-mail address: huang@iseis.org (G. H. Huang).

ISSN: $1726-2135$ print/1684-8799 online

(C) 2012 ISEIS All rights reserved. doi:10.3808/jei.201200203
2007, 2008; Nie et al., 2007; Lv et al., 2010; Sun and Huang, 2010; Yan et al., 2010; Fan et al., 2009, 2012a, 2012b). Among them, IMP was an effective approach in dealing with uncertainties, since it does not require distributional information for input parameters (Tong, 1994; Chinneck and Ramada, 2000; Sengupta et al., 2001; Fiedler et al., 2006; Oliveira and Antunes, 2007). Huang et al. (1992) proposed an interval linear programming (ILP) method through incorporating interval numbers into a linear programming (LP) framework and applied it to solid waste management. After that, many inexact LP methods were proposed for dealing with a variety of uncertainties in environmental management problems (Nie et al., 2007; He et al., 2008; Liu et al., 2008).

The two-step method (TSM) proposed by Huang et al. (1992) was mostly used to solve IMP problems. In this method, an ILP problem was converted into two sub-problems that correspond to lower and upper bounds of its objective-function value (Huang et al., 1992). TSM is effective in solving ILP problems without significantly increased computational requirements. However, the main disadvantage of TSM is the potential existence of constraint violation in its solution space. Consequently, Zhou et al. (2008) and Huang and Cao (2011) improved the algorithm of Huang et al. (1992). In detail, Zhou et al. (2008) developed a modified interval linear programming (MILP) method, through incorporating additional constraints into the lower- and upper-bound submodels. Cao and Huang (2011) proposed a three-step method (ThSM) for solving the ILP problem; following a feasibility test, a constricting process for the decision space was implemented, based on the original space from the TSM solutions. The MILP and ThSM could thus help avoid constraint violation in the ILP solution space. However, they were based on relatively harsh constraints that 
led to significantly shrunk decision space, resulting in loss of many feasible decision alternatives. In addition, ThSM might also be associated with issues of infeasibility in the solution process (particularly when the mid-value TSM solutions are infeasible), as well as significantly increased computation requirement. Therefore, a more effective algorithm is desired.

The objective of this study is to develop a robust two-step method (RTSM) for solving ILP problems. The RTSM can help at least guarantee feasibility of the generated solutions under the best-case constraints, while the dimensions of the decision space are not significantly compromised. This is meaningful since, within such a space, all decision alternatives generated through various trade-off analyses will be guaranteed to be potentially feasible points with different feasibility levels. The RTSM also possesses the advantage of convenience for practical applications, since it does not lead to more complicated solution procedures. Finally, a simplified example will be developed to illustrate the solution process.

\section{Review of the Existing Solution Methods for ILP}

According to Huang (1998), an ILP model can be presented as follows:

$\operatorname{Max} f^{ \pm}=C^{ \pm} X^{ \pm}$

subject to:

$A^{ \pm} X^{ \pm} \leq B^{ \pm}$

$X^{ \pm} \geq 0$

where $A^{ \pm} \in\left\{R^{ \pm}\right\}^{m \times n}, \mathrm{C}^{ \pm} \in\left\{R^{ \pm}\right\}^{1 \times n}, B^{ \pm} \in\left\{R^{ \pm}\right\}^{m \times 1}, X^{ \pm} \in\left\{R^{ \pm}\right\}^{n \times 1}$; $R^{ \pm}$denotes a set of interval numbers; $A^{ \pm}=\left(a_{i j}^{ \pm}\right)_{m \times n}, C^{ \pm}=$ $\left(c_{1}^{ \pm}, c_{2}^{ \pm}, \ldots, c_{n}^{ \pm}\right), B^{ \pm}=\left(b_{1}^{ \pm}, b_{2}^{ \pm}, \ldots, b_{m}^{ \pm}\right)^{T}$ and $X^{ \pm}=\left(x_{1}^{ \pm}, x_{2}^{ \pm}, \ldots, x_{n}^{ \pm}\right)^{T}$. An interval number $\left(a^{ \pm}\right)$is defined as (Huang et al., 1992): $a^{ \pm}=\left[a^{-}, a^{+}\right]=\left\{t \in a \mid a^{-} \leq t \leq a^{+}\right\}$.

To solve model (1), Huang et al. (1992) proposed a twostep method (TSM) through analyzing interrelationships among parameters and variables in the objective and constraints. The main idea of TSM is to convert the original ILP model into two LP submodels which correspond to the lower and upper bounds of the objective-function value. In detail, for $n$ interval coefficients $c_{j}^{ \pm}(j=1,2, \ldots, n)$ in the objective function, the former $k$ coefficients are assumed to be positive (i.e. $c_{j}^{ \pm} \geq 0$, for $j=1,2, \ldots, k)$, and the latter $(n-k)$ coefficients are negative (i.e. $c_{j}^{ \pm} \leq 0$, for $\left.j=k+1, k+2, \ldots, n\right)$. Thus the first submodel of model (1) would correspond to $f^{+}$. It can be formulated as follows (assume that $b_{i}^{ \pm}>0$ and $f^{ \pm}>0$ ):

$\operatorname{Max} f^{+}=\sum_{j=1}^{k} c_{j}^{+} x_{j}^{+}+\sum_{j=k+1}^{n} c_{j}^{+} x_{j}^{-}$

subject to:

$$
\sum_{j=1}^{k}\left|a_{i j}^{ \pm}\right|^{-} \operatorname{Sign}\left(a_{i j}^{ \pm}\right) x_{j}^{+}+\sum_{j=k+1}^{n}\left|a_{i j}^{ \pm}\right|^{+} \operatorname{Sign}\left(a_{i j}^{ \pm}\right) x_{j}^{-} \leq b_{i}^{+}, i=1,2, \ldots, m .
$$

$x_{j}^{+} \geq 0, j=1,2, \ldots, k$

$x_{j}^{-} \geq 0, j=k+1, k+2, \ldots, n$

Solutions of $x_{\text {jopt }}^{+}(j=1,2, \ldots, k)$ and $x_{\text {jopt }}^{-} \quad(j=k+1, k$ $+2, \ldots, n)$ can be obtained through solving submodel (2). Based on the solutions of model (2), the submodel corresponding to $f^{-}$can be formulated as follows (assume that $b_{i}^{ \pm}>0$ and $\left.f^{ \pm}>0\right)$ :

$\operatorname{Max} f^{-}=\sum_{j=1}^{k} c_{j}^{-} x_{j}^{-}+\sum_{j=k+1}^{n} c_{j}^{-} x_{j}^{+}$,

subject to:

$\sum_{j=1}^{k}\left|a_{i j}^{ \pm}\right|^{+} \operatorname{Sign}\left(a_{i j}^{ \pm}\right) x_{j}^{-}+\sum_{j=k+1}^{n}\left|a_{i j}^{ \pm}\right|^{-} \operatorname{Sign}\left(a_{i j}^{ \pm}\right) x_{j}^{+} \leq b_{i}^{-}, i=1,2, \ldots, m$.

$0 \leq x_{j}^{-} \leq x_{j o p t}^{+}, j=1,2, \ldots, k$.

$x_{j}^{+} \geq x_{j o p t}^{-}, j=k+1, k+2, \ldots, n$.

From model (3), solutions of $x_{\text {jopt }}^{-}(j=1,2, \ldots, k)$ and $x_{j o p t}^{+}(j=k+1, k+2, \ldots, n)$ can be obtained. Thus, the final solutions of $f_{\text {opt }}^{ \pm}=\left[f_{\text {opt }}^{-}, f_{\text {opt }}^{+}\right]$and $x_{j o p t}^{ \pm}=\left[x_{\text {jopt }}^{-}, x_{\text {jopt }}^{+}\right]$can be obtained for model (1).

The TSM has been widely used for solving many inexact environmental management problems that can be formulated as ILP models (Huang et al., 1995). However, its main weakness is the potential violation of the best-case constraints when the detailed decision point varies within the generated decision space. For example, consider an ILP model: $\max \left\{c_{1}^{ \pm} x_{1}^{ \pm}-c_{2}^{ \pm} x_{2}^{ \pm} \mid\right.$ $\left.a_{11}^{ \pm} x_{1}^{ \pm}+a_{12}^{ \pm} x_{2}^{ \pm} \leq b_{1}^{ \pm}, a_{21}^{ \pm} x_{1}^{ \pm}+a_{22}^{ \pm} x_{2}^{ \pm} \leq b_{2}^{ \pm}\right\}$, and assume that all of its coefficients are non-negative. According to TSM, constraints $a_{i 1}^{+} x_{1}^{-}+a_{i 2}^{-} x_{2}^{+} \leq b_{i}^{-}$and $a_{i 1}^{-} x_{1}^{+}+a_{i 2}^{+} x_{2}^{-} \leq b_{i}^{+}$correspond to the lower and upper bounds of the objective-function value, respectively. However, these two constraints cannot fully guarantee feasibility of the best-case constraints (i.e. $a_{i 1}^{-} x_{1}+a_{i 2}^{-} x_{2} \leq b_{i}^{+}$) for any $x_{1} \in\left[x_{1}^{-}, x_{1}^{+}\right]$and $x_{2} \in\left[x_{2}^{-}, x_{2}^{+}\right]$.

Zhou et al. (2008) modified the TSM algorithm through introducing additional constraints into the lower- and upperbound submodels. For model (1), their modified ILP (or MILP) solution procedures are presented as follows:

Submodel 1

$\operatorname{Max} f^{+}=\sum_{j=1}^{k} c_{j}^{+} x_{j}^{+}+\sum_{j=k+1}^{n} c_{j}^{+} x_{j}^{-}$ 
subject to:

$\sum_{j=1}^{k}\left|a_{i j}^{ \pm}\right|^{-} \operatorname{Sign}\left(a_{i j}^{ \pm}\right) x_{j}^{+}+\sum_{j=k+1}^{n}\left|a_{i j}^{ \pm}\right|^{+} \operatorname{Sign}\left(a_{i j}^{ \pm}\right) x_{j}^{-} \leq b_{i}^{+}, i=1,2, \ldots, m$.

$x_{j}^{+} \geq 0, j=1,2, \ldots, k$

$x_{j}^{-} \geq 0, j=k+1, k+2, \ldots, n$

Submodel 2

$\operatorname{Max} f^{-}=\sum_{j=1}^{k} c_{j}^{-} x_{j}^{-}+\sum_{j=k+1}^{n} c_{j}^{-} x_{j}^{+}$,

subject to:

$\sum_{j=1}^{k}\left|a_{i j}^{ \pm}\right|^{+} \operatorname{Sign}\left(a_{i j}^{ \pm}\right) x_{j}^{-}+\sum_{j=k+1}^{n}\left|a_{i j}^{ \pm}\right|^{-} \operatorname{Sign}\left(a_{i j}^{ \pm}\right) x_{j}^{+} \leq b_{i}^{-}, i=1,2, \ldots, m$.

$\sum_{j=k-p+1}^{k}-\left(\left|a_{\delta j}^{ \pm}\right|^{+} x_{j}^{-}-\left|a_{\delta j}^{ \pm}\right|^{-} x_{j o p t}^{+}\right)+\sum_{j=n-q+1}^{n}\left(\left|a_{\delta j}^{ \pm}\right|^{-} x_{j}^{+}-\left|a_{\delta j}^{ \pm}\right|^{+} x_{j o p t}^{-}\right) \leq 0$

$0 \leq x_{j}^{-} \leq x_{j o p t}^{+}, j=1,2, \ldots, k$.

$x_{j}^{+} \geq x_{j o p t}^{-}, j=k+1, k+2, \ldots, n$.

where $\delta$ is the number of constraints in Equation (4b) that meet $\sum_{j=1}^{k}\left|a_{\delta j}^{ \pm}\right| \operatorname{Sign}\left(a_{\delta j}^{ \pm}\right) x_{j o p t}^{+}+\sum_{j=k+1}^{n}\left|a_{\delta j}^{ \pm}\right|^{+} \operatorname{Sign}\left(a_{\delta j}^{ \pm}\right) x_{j o p t}^{-}=b_{\delta}^{+}$and $a_{\delta j}^{ \pm} \leq 0$ for $j=k-p+1, k-p+2, \ldots, k$ and $a_{\delta j}^{ \pm} \geq 0$ for $j=n$ $-q+1, n-q+2, \ldots, n$.

The MILP method can ensure the ILP solution space to be absolutely feasible, without violation of the best-case constraints. However, the MILP was based on relatively harsh constraints (i.e. additional constraints to submodel 5) that led to significantly shrunk decision space, resulting in loss of many feasible decision alternatives; moreover, uncertainty of the objective function may be enlarged due to the additional constraints in submodel (5).

Cao and Huang (2011) proposed a three-step method (ThSM) for solving the ILP problem; following a feasibility test, a constricting process for the decision space was implemented, based on the original space from the TSM solutions. The ThSM consisted of the following steps (Cao and Huang, 2011):

Step 1: generate solutions of model (1) through TSM.

Step 2: conduct feasibility test to analyze whether the TSM solutions can satisfy the best-case constraints. For constraint $i$ $(i=1,2, \ldots, m)$, the idea of feasibility test is to identify $X^{*} \in$ $X_{\text {opt }}^{ \pm}$such that $\sum_{j=1}^{n} a_{i j}^{-} x_{j}^{*}=\max \left\{\sum_{j=1}^{n} a_{i j}^{-} x_{j} \mid x_{j} \in x_{j}^{ \pm}\right\}$; if $\sum_{j=1}^{n} a_{i j}^{-} x_{j}^{*} \leq b_{i}^{+}$is tenable, then $X_{o p t}^{ \pm}$pass the feasibility test; otherwise, it means that part of solutions from $X_{\text {opt }}^{ \pm}$will violate the constraints.

Step 3: eliminate the infeasible solution zone through a constricting approach. For solutions that do not pass the feasibility test, two constricting approaches are proposed to remove them:

\section{ThSM-I}

$\operatorname{Max} q$

subject to:

$\sum_{j=1}^{p_{i}} a_{i j}^{-} q d_{j}-\sum_{j=p_{i}+1}^{n_{0}} a_{i j}^{-} q d_{j} \leq b_{i}^{+}-\sum_{j=1}^{n} a_{i j}^{-} m_{j}, i=1,2, \ldots, m$.

$0 \leq q \leq 1$

ThSM-II

$\operatorname{Max} q_{1} \times q_{2} \times \ldots \times q_{n_{0}}$

subject to:

$$
\begin{aligned}
& \sum_{j=1}^{p_{i}} a_{i j}^{-} q_{j} d_{j}-\sum_{j=p_{i}+1}^{n_{0}} a_{i j}^{-} q_{j} d_{j} \leq b_{i}^{+}-\sum_{j=1}^{n} a_{i j}^{-} m_{j}, i=1,2, \ldots, m . \\
& 0 \leq q_{j} \leq 1
\end{aligned}
$$

where $m_{j}=0.5\left(x_{j o p t}^{-}+x_{j o p t}^{+}\right) ; d_{j}=0.5\left(x_{j o p t}^{+}-x_{j o p t}^{-}\right) ; j=1,2, \ldots$, $n_{0} ; n_{0}$ means the number of interval solutions obtained through submodels (2) and (3).

The main advantage of the ThSM method is its ability to avoid constraint violation in the ILP solution space. However, many feasible solutions will be eliminated in the constricting process which compresses the solution space from all dimensions. Particularly, it leads to loss of the solution zones that are close to the worst-case constraints. In addition, ThSM might also be associated with issues of infeasibility in the solution process. For example, when the mid-value of TSM solutions [i.e. the solution set defined by $x_{j}^{m}=0.5\left(x_{j o p t}^{-}+x_{j o p t}^{+}\right), \forall j$ ] does not pass feasibility test, then models (6) and (7) will become infeasible. Also, ThSM may lead to significantly increased computation requirement, which may hinder its practical applications, especially for problems with a large number of constraints.

\section{Robust Two-Step Method}

A robust two-step method (RTSM) is proposed for solving ILP problems, where the best-cases constraints of model (1) will be incorporated within the submodel corresponding to $f^{+}$ (when the objective is to be maximized). This can thus help 
avoid absolute constraint violation (i.e. violations even beyond the best-case constraints) as the decision variables fluctuated within the generated decision space.

Lemma 1 (Huang et al. 1995): For $A \in\left[A^{-}, A^{+}\right]$and $B$ $\in\left[B^{-}, B^{+}\right]$, denoting $Q=\{X \mid A X \leq B, X \geq 0\}, Q^{-}=\left\{X \mid A^{+} X\right.$ $\left.\leq B^{-}, X \geq 0\right\}$, and $Q^{+}=\left\{X \mid A^{-} X \leq B^{+}, X \geq 0\right\}$, we have $Q^{+} \supseteq$ $Q \supseteq Q^{-}$:

Proof: If both $X \in Q^{-}$and $X \geq 0$ hold, we have $A X \leq A^{+} X$ $\leq B^{-} \leq B$, such that $X \in Q$ holds. Furthermore, if both $X \in Q$ and $X \geq 0$ hold, we have $A^{-} X \leq A X \leq B \leq B^{+}$, such that $X \in Q^{+}$ holds. Hence, $Q^{+} \supseteq Q \supseteq Q^{-}$

Remark 1: Lemma 1 provides the relationships between feasible $X$ sets under two extreme constraints (best and worst cases).

Lemma 2(Huang et al., 1995): For $n$ interval coefficients $c_{j}^{ \pm}(j=1,2, \ldots, n)$ in the objective function of model (1), if $k_{1}$ of them are positive, and $k_{2}$ are negative, let the former $k_{1}$ coefficients be positive, i.e. $c_{j}^{ \pm} \geq 0\left(j=1,2, \ldots, k_{1}\right)$ and the latter $k_{2}$ coefficients be negative, i.e. $c_{j}^{ \pm} \leq 0\left(j=k_{1}+1, k_{1}+2, \ldots, n\right)$, where $k_{1}+k_{2}=n$ (the model does not include the situation in which the two bounds of $c_{j}^{ \pm}$have different signs). Thus, the following expression can be developed for the lower and upper bounds of $f^{ \pm}$:

$$
\begin{array}{r}
f^{+}=\sum_{j=1}^{k_{1}} c_{j}^{+} x_{j}^{+}+\sum_{j=k_{1}+1}^{n} c_{j}^{+} x_{j}^{-} \\
f^{-}=\sum_{j=1}^{k_{1}} c_{j}^{-} x_{j}^{-}+\sum_{j=k_{1}+1}^{n} c_{j}^{-} x_{j}^{+}
\end{array}
$$

Proof: Straightforward.

Corollary 1: the largest feasible area corresponding to $f^{+}$ can be formed as follows:

$Q_{\max }^{+}=\left\{x_{j}^{+} \geq 0, j=1,2, \ldots, k_{1} ; x_{j}^{-} \geq 0, j=k_{1}+1, k_{1}+2, \ldots, n ; \mid \sum_{j=1}^{k_{1}}\right.$

$\left.a_{i j}^{-} x_{j}^{+}+\sum_{j=k_{1}+1}^{n} a_{i j}^{-} x_{j}^{-} \leq b_{i}^{+}, \forall i\right\}$

Proof: Assume $a_{i j}^{u} \in\left[a_{i j}^{-}, a_{i j}^{+}\right]$and $Q_{u}^{+}=\left\{x_{j}^{+} \geq 0, j=1,2, \ldots\right.$, $k_{1} ; \quad x_{j}^{-} \geq 0, j=k_{1}+1, k_{1}+2, \ldots, n \mid \sum_{j=1}^{k_{1}} a_{i j}^{u} x_{j}^{+}+\sum_{j=k_{1}+1}^{n} a_{i j}^{u} x_{j}^{-} \leq$ $\left.b_{i}^{+}, \forall i\right\}$. For any $X_{0}=\left(x_{j 0}^{+} \geq 0, j=1,2, \ldots, k_{1} ; x_{j 0}^{-} \geq 0, j=k_{1}\right.$ $\left.+1, k_{1}+2, \ldots, n\right) \in Q_{u}^{+}, \sum_{j=1}^{k_{1}} a_{i j}^{u} x_{j 0}^{+}+\sum_{j=k_{1}+1}^{n} a_{i j}^{u} x_{j 0}^{-} \leq b_{i}^{+}$holds. Because $a_{i j}^{-} \leq a_{i j}^{u} \leq a_{i j}^{+}$and $x_{j 0}^{ \pm} \geq 0$, we have $\sum_{j=1}^{k_{1}} a_{i j}^{-} x_{j 0}^{+}+$ $\sum_{j=k_{1}+1}^{n} a_{i j}^{-} x_{j 0}^{-} \leq \sum_{j=1}^{k_{1}} a_{i j}^{u} x_{j 0}^{+}+\sum_{j=k_{1}+1}^{n} a_{i j}^{u} x_{j 0}^{-} \leq b_{i}^{+}, \forall i$. Therefore, $X_{0} \in Q_{\max }^{+}$, namely $Q_{u}^{+} \subseteq Q_{\max }^{+}$. $f^{-}$is :

Corollary 2: the smallest feasible area corresponding

$Q_{\text {min }}^{-}=\left\{x_{j}^{-} \geq 0, j=1,2, \ldots, k_{1} ; x_{j}^{+} \geq 0, j=k_{1}+1, k_{1}+2, \ldots, n ; \mid \sum_{j=1}^{k_{1}}\right.$ $\left.a_{i j}^{+} x_{j}^{-}+\sum_{j=k_{1}+1}^{n} a_{i j}^{+} x_{j}^{+} \leq b_{i}^{-}\right\}$

Proof: Assume $a_{i j}^{l} \in\left[a_{i j}^{-}, a_{i j}^{+}\right]$and $Q_{l}^{-}=\left\{x_{j}^{-} \geq 0, j=1,2, \ldots\right.$, $k_{1} ; x_{j}^{+} \geq 0, j=k_{1}+1, k_{1}+2, \ldots, n \mid \sum_{j=1}^{k_{1}} a_{i j}^{l} x_{j}^{-}+\sum_{j=k_{1}+1}^{n} a_{i j}^{l} x_{j}^{+} \leq$ $\left.b_{i}^{-}, \forall i\right\}$. Consider $\forall X=\left(x_{j}^{-} \geq 0, j=1,2, \ldots, k_{1} ; x_{j}^{+} \geq 0, j=\right.$ $\left.k_{1}+1, k_{1}+2, \ldots, n\right) \in Q_{\min }^{-}$, then $\sum_{j=1}^{k_{1}} a_{i j}^{+} x_{j}^{-}+\sum_{j=k+1}^{n} a_{i j}^{+} x_{j}^{+} \leq$ $b_{i}^{+}$holds. Since $a_{i j}^{-} \leq a_{i j}^{l} \leq a_{i j}^{+}$and $x_{j}^{ \pm} \geq 0$, we have $\sum_{j=1}^{j+k_{1} l j} a_{i j}^{l} x_{j}^{-}$ $+\sum_{j=k_{1}+1}^{n} a_{i j}^{l} x_{j}^{+} \leq \sum_{j=1}^{k_{1}} a_{i j}^{+} x_{j}^{-}+\sum_{j=k_{1}+1}^{n} a_{i j}^{+} x_{j}^{+} \leq b_{i}^{-}, \forall i$. Therefore, $X \in Q_{l}^{-}$, namely $Q_{\text {min }}^{-} \subseteq Q_{l}^{-}$.

Theorem 1: (Huang et al., 1995) In order to obtain interval solutions expressed as $f_{\text {opt }}^{ \pm}=\left[f_{\text {opt }}^{-}, f_{\text {opt }}^{+}\right]$and $x_{\text {jopt }}^{ \pm}=\left[x_{\text {jopt }}^{-}\right.$, $x_{\text {jopt }}^{+}$, constraints corresponding to $f^{+}$can be developed as follows:

$\sum_{j=1}^{k}\left|a_{i j}^{ \pm}\right|^{-} \operatorname{Sign}\left(a_{i j}^{ \pm}\right) x_{j}^{+}+\sum_{j=k+1}^{n}\left|a_{i j}^{ \pm}\right|^{+} \operatorname{Sign}\left(a_{i j}^{ \pm}\right) x_{j}^{-} \leq b_{i}^{+}, i=1,2, \ldots, m$

Similarly, the relevant constraints corresponding to $f$ can also be developed as follows:

$\sum_{j=1}^{k}\left|a_{i j}^{ \pm}\right|^{+} \operatorname{Sign}\left(a_{i j}^{ \pm}\right) x_{j}^{-}+\sum_{j=k+1}^{n}\left|a_{i j}^{ \pm}\right|^{-} \operatorname{Sign}\left(a_{i j}^{ \pm}\right) x_{j}^{+} \leq b_{i}^{-}, i=1,2, \ldots, m$

Lemma 3: Consider constraint $i$ in equation (1b), $\sum_{j=1}^{n} a_{i j}^{ \pm} x_{j}^{ \pm} \leq b_{i}^{ \pm}$, where $a_{i j}^{ \pm} \geq 0 \quad\left(j=1,2, \ldots, l_{i 1}\right)$ and $a_{i j}^{ \pm}$ $\leq 0\left(j=l_{i 1}+1, l_{i 1}+2, \ldots, n\right)$. For any $x_{j} \in\left[x_{j}^{-}, x_{j}^{+}\right](j=1,2, \ldots$, $n$ ), the best-case constraint of constraint $i$ (i.e. $\sum_{j=1}^{n} a_{i j}^{-} x_{j} \leq b_{i}^{+}$) can be satisfied, if and only if:

$\sum_{j=1}^{l_{i 1}} a_{i j}^{-} x_{j}^{+}+\sum_{j=l_{i 1}+1}^{n} a_{i j}^{-} x_{j}^{-} \leq b_{i}^{+}$

Proof: Since $a_{i j}^{ \pm} \geq 0 \quad\left(j=1,2, \ldots, l_{i 1}\right)$ and $a_{i j}^{ \pm} \leq 0 \quad(j=$ $\left.l_{i 1}+1, l_{i 1}+2 \ldots, n\right)$, then for any $x_{j} \in\left[x_{j}^{-}, x_{j}^{+}\right] \geq 0(j=1,2, \ldots$, $n), x_{j} \leq x_{j}^{+}$and $x_{j} \geq x_{j}^{-}$hold. Thus we have $a_{i j}^{-} x_{j} \leq a_{i j}^{-} x_{j}^{+}$ $\left(j=1,2, \ldots, l_{i 1}\right)$ and $a_{i j}^{-} x_{j} \leq a_{i j}^{-} x_{j}^{-}\left(j=l_{i 1}+1, l_{i 1}+2 \ldots, n\right)$. If $\sum_{j=1}^{l_{i 1}} a_{i j}^{-} x_{j}^{+}+\sum_{j=l_{i 1}+1}^{n} a_{i j}^{-} x_{j}^{-} \leq b_{i}^{+}$, then we have $\sum_{j=1}^{l_{i 1}} a_{i j}^{-} x_{j}+$
$\sum_{j=l_{i 1}+1}^{n} a_{i j}^{-} x_{j} \leq b_{i}^{+}$.

Theorem 2: Consider constraint $i_{0}$ of model (1):

$\sum_{j=1}^{k_{1}} a_{i_{0} j}^{ \pm} x_{j}^{ \pm}+\sum_{j=k_{1}+1}^{n} a_{i_{0} j}^{ \pm} x_{j}^{ \pm} \leq b_{i_{0}}^{ \pm}$

Three criteria exist for determining whether additional constraints are needed to avoid violation of the best-case constraint:

(1) If $a_{i_{0} j}^{ \pm} \times c_{j}^{ \pm} \geq 0 \forall j$, then solution $x_{j o p t}^{ \pm}=\left[x_{j o p t}^{-}, x_{\text {jopt }}^{+}\right]$ obtained from submodels (2) and (3) can ensure that for any $x_{j}$ $\in\left[x_{\text {jopt }}^{-}, x_{\text {jopt }}^{+}\right](j=1,2, \ldots, n), \sum_{j=1}^{k_{1}} a_{i_{0} j}^{-} x_{j}+\sum_{j=k_{1}+1}^{n} a_{i_{0} j}^{-} x_{j}$ $\leq b_{i_{0}}^{+}$is satisfied. 
(2) If $a_{i_{0} j}^{ \pm} \times c_{j}^{ \pm} \leq 0 \forall j$, then the solutions $x_{\text {jopt }}^{ \pm}=\left[x_{\text {jopt }}^{-}, x_{\text {jopt }}^{+}\right]$ obtained through submodels (2) and (3) can guarantee that, for any $x_{j} \in\left[x_{\text {jopt }}^{-}, x_{j o p t}^{+}\right](j=1,2, \ldots, n), \sum_{j=1}^{k_{1}} a_{i_{0} j}^{-} x_{j}+$ $\sum_{j=k_{1}+1}^{n} a_{i_{0} j}^{-} x_{j} \leq b_{i_{0}}^{+}$is satisfied.

(3) If $a_{i_{0} j}^{ \pm} \times c_{j}^{ \pm} \geq 0\left[j=1,2, \ldots, l_{i_{0} 1}\left(l_{i_{0} 1} \leq k\right)\right.$ and $k+1, k$ $\left.+2 \ldots, l_{i_{0} 2}\left(l_{i_{0} 2}>k\right)\right]$ and $a_{i_{0} j}^{ \pm} \times c_{j}^{ \pm} \leq 0\left[j=l_{i_{0} 1}+1, l_{i_{0} 1}+2, \ldots\right.$, $k$ and $\left.l_{i_{0} 2}+1, l_{i_{0} 2}+2, \ldots, n\right]$, then solutions $x_{\text {jopt }}^{ \pm}=\left[x_{\text {jopt }}^{-}, x_{\text {jopt }}^{+}\right]$ obtained from submodels (2) and (3) will not lead to violation of the best-case constraint $\left(\sum_{j=1}^{n} a_{i j}^{-} x_{j} \leq b_{i}^{+}\right)$, if and only if:

$\sum_{j=1}^{l_{i_{0} 1}} a_{i_{0} j}^{-} x_{j}^{+}+\sum_{j=l_{i_{0}}+1}^{k} a_{i_{0} j}^{-} x_{j o p t}^{-}+\sum_{j=k+1}^{l_{i_{0} 2}} a_{i_{0} j}^{-} x_{j}^{-}+\sum_{j=l_{i_{0} 2}+1}^{n} a_{i_{0} j}^{-} x_{j o p t}^{+}$

$\leq b_{i_{0}}^{+}$

where $x_{j o p t}^{-}$and $x_{j o p t}^{+}$are solutions obtained from submodel (3) which corresponds to $f^{-}$.

\section{Proof:}

(1) From model (2), we have: $\sum_{j=1}^{k}\left|a_{i_{0} j}^{ \pm}\right|^{-} \operatorname{Sign}\left(a_{i_{0} j}^{ \pm}\right) x_{j}^{+}+$ $\sum_{j=k+1}^{n}\left|a_{i_{0} j}^{ \pm}\right|^{+} \operatorname{Sign}\left(a_{i_{0} j}^{ \pm}\right) x_{j}^{-} \leq b_{i_{0}}^{+}$. Since $a_{i_{0} j}^{ \pm} \times c_{j}^{ \pm} \geq 0 \forall j$, we have: $a_{i_{0} j}^{ \pm} \geq 0(j=1,2, \ldots, k)$ and $a_{i_{0} j}^{ \pm} \leq 0(j=k+1, k+2, \ldots$, $n)$. Thus, $\left|a_{i_{0} j}^{ \pm}\right|^{-} \operatorname{Sign}\left(a_{i_{0} j}^{ \pm}\right) x_{j}^{+}=a_{i_{0} j}^{-} x_{j}^{+}$, and $\left|a_{i_{0} j}^{ \pm}\right|^{+} \operatorname{Sign}\left(a_{i_{0} j}^{ \pm}\right) x_{j}^{-}$ $=a_{i_{0} j}^{-} x_{j}^{-}$hold. Therefore, we have $\sum_{j=1}^{k}\left|a_{i_{0} j}^{ \pm}\right|^{-} \operatorname{Sign}\left(a_{i_{0} j}^{ \pm}\right) x_{j}^{+}+$ $\sum_{j=k+1}^{n}\left|a_{i_{0} j}^{ \pm}\right|^{+} \operatorname{Sign}\left(a_{i_{0} j}^{ \pm}\right) x_{j}^{-}=\sum_{j=1}^{k} a_{i_{0} j}^{-} x_{j}^{+}+\sum_{j=k+1}^{n} a_{i_{0} j}^{-} x_{j}^{-} \leq b_{i_{0}}^{+}$. For any $x_{j} \in\left[x_{j}^{-}, x_{j}^{+}\right](j=1,2, \ldots, n), a_{i_{0} j}^{-} x_{j} \leq a_{i_{0}}^{-} x_{j}^{+} \quad(j=1$, $2, \ldots, k)$ and $a_{i_{j}}^{-} x_{j} \leq a_{i_{j}}^{-} x_{j}^{-} \quad(j=k+1, k+2, \ldots, n)$ hold. Consequently, we have $\sum_{j=1}^{i_{i_{0} j} j} a_{j}^{-}+\sum_{j=k+1}^{n} a_{i_{0} j}^{-} x_{j} \leq \sum_{j=1}^{k} a_{i_{0} j}^{-} x_{j}^{+}$ $+\sum_{j=k+1}^{n} a_{i_{0} j}^{-} x_{j}^{-} \leq b_{i_{0}}^{+}$.

(2) Based on model (3), $\sum_{j=1}^{k}\left|a_{i_{0} j}^{ \pm}\right|^{+} \operatorname{Sign}\left(a_{i_{0} j}^{ \pm}\right) x_{j}^{-}+\sum_{j=k+1}^{n}$ $\left|a_{i_{0} j}^{ \pm}\right| \operatorname{Sign}\left(a_{i_{0} j}^{ \pm}\right) x_{j}^{+} \leq b_{i_{0}}^{-}$holds. Since $a_{i_{0} j}^{ \pm} \times c_{j}^{ \pm} \leq 0 \forall j$, then we have $a_{i_{0} j}^{ \pm} \leq 0(j=1,2, \ldots, k)$ and $a_{i_{0} j}^{ \pm} \geq 0(j=k+1, k$ $+2 \ldots, n)$. Thus, $\left|a_{i_{0} j}^{ \pm}\right|^{+} \operatorname{Sign}\left(a_{i_{0} j}^{ \pm}\right) x_{j}^{-}=a_{i_{0} j}^{-} x_{j}^{-}$and $\left|a_{i_{0} j}^{ \pm}\right|^{-}$ $\operatorname{Sign}\left(a_{i_{0} j}^{ \pm}\right) x_{j}^{+}=a_{i_{0} j}^{-} x_{j}^{+}$hold. Consequently, we have $\sum_{j=1}^{k}$ $\left|a_{i_{0} j}^{ \pm}\right|^{+} \operatorname{Sign}\left(a_{i_{0} j}^{ \pm}\right) x_{j}^{-}+\sum_{j=k+1}^{n}\left|a_{i_{0} j}^{ \pm}\right|^{-} \operatorname{Sign}\left(a_{i_{0} j}^{ \pm}\right) x_{j}^{+}=\sum_{j=1}^{k} a_{i_{0} j}^{-} x_{j}^{-}+$ $\sum_{j=k+1}^{n} a_{i_{0} j}^{-} x_{j}^{+} \leq b_{i_{0}}^{-} \leq b_{i_{0}}^{+}$. For any $x_{j} \in\left[x_{j}^{-}, x_{j}^{+}\right](j=1$, $2, \ldots, n), a_{i_{0} j}^{-} x_{j} \leq a_{i_{0} j}^{-} x_{j}^{-}(j=1,2, \ldots, k)$ and $a_{i_{0} j}^{-} x_{j} \leq a_{i_{0} j}^{-} x_{j}^{+}$ $(j=k+1, k+2, \ldots, n)$ hold. Therefore, we have $\sum_{j=1}^{k} a_{i_{0}}^{-} x_{j}$ $+\sum_{j=k+1}^{n} a_{i_{0} j}^{-} x_{j} \leq \sum_{j=1}^{k} a_{i_{0} j}^{-} x_{j}^{-}+\sum_{j=k+1}^{n} a_{i_{0} j}^{-} x_{j}^{+} \leq b_{i_{0}}^{-} \leq b_{i_{0}}^{+}$.

(3) If $a_{i_{0} j}^{ \pm} \times c_{j}^{ \pm} \geq 0\left[j=1,2, \ldots, l_{i_{0} 1}\left(l_{i_{0} 1} \leq k\right)\right.$ and $k+1, k+$ $\left.2, \ldots, l_{i_{0} 2}\left(l_{i_{0} 2}>k\right)\right]$ and $a_{i_{0} j}^{ \pm} \times c_{j}^{ \pm} \leq 0\left[j=l_{i_{0} 1}+1, l_{i_{0} 1}+2, \ldots, k\right.$ and $\left.l_{i_{0} 2}+1, l_{i_{0} 2}+2, \ldots, n\right]$, we have $a_{i_{0} j}^{ \pm} \geq 0\left(j=1,2, \ldots, l_{i_{0} 1}\right.$ and $\left.l_{i_{0} 2}+1, l_{i_{0} 2}+2, \ldots, n\right)$ and $a_{i_{0} j}^{ \pm} \leq 0\left(j=l_{i_{0} 1}+1, l_{i_{0} 2}+2, \ldots, k, k\right.$ $\left.+1, \ldots, l_{i_{0} 2}\right)$. When equation (13) holds, for any $x_{j} \in\left[x_{j}^{-}, x_{j}^{+}\right](j$ $=1,2, \ldots, n)$ and $x_{j}^{-} \leq x_{j} \leq x_{j}^{+}$, we have $\sum_{j=1}^{l_{i_{0}} 1} a_{i_{0} j}^{-} x_{j}^{+} \geq \sum_{j=1}^{l_{i_{1}}} a_{i_{0} j}^{-} x_{j}$ and $\sum_{j=l_{i}+1}^{n} a_{i_{0} j}^{-} x_{j o p t}^{+} \geq \sum_{j=l_{j}+1}^{n} a_{i_{0} j}^{-} x_{j}$ due to $a_{i_{0} j}^{ \pm} \geq 0 \quad(j=1$, $2, \ldots, l_{i_{0} 1}$ and $\left.l_{i_{0} 2}+1, l_{i_{0} 2}^{j+l^{2}} 2_{2}, \ldots, n\right)$; meanwhile $\sum_{j=l_{i_{1}+1}+1}^{k} a_{i_{0} j}^{-}$ $x_{j o p t}^{-} \geq \sum_{j=l_{j, 1}+1}^{k} a_{i_{0} j}^{-} x_{j}$ and $\sum_{j=k+1}^{l_{i_{0} 2}} a_{i_{0} j}^{-} x_{j}^{-} \geq \sum_{j=k+1}^{l_{i_{0} 2}} a_{i_{0} j}^{-} x_{j}$ will hold since $a_{i_{0} j}^{ \pm} \leq 0\left(j=l_{i_{0} 1}+1, l_{i_{0} 1}+2, \ldots, k, k+1, \ldots, l_{i_{0} 2}\right)$. Therefore, we have $\sum_{j=1}^{n} a_{i_{0} j}^{-} x_{j} \leq \sum_{j=1}^{l_{i_{0}} 1} a_{i_{0} j}^{-} x_{j}^{+}+\sum_{j=l_{i_{0}}+1}^{k} a_{i_{0} j}^{-} x_{j o p t}^{-}+\sum_{j=k+1}^{l_{i_{0} 2}}$ $a_{i_{0} j}^{-} x_{j}^{-}+\sum_{j=l_{i_{0}}+1}^{n} a_{i_{0} j}^{-} x_{j o p t}^{+} \leq b_{i_{0}}^{+}$.
Remark 3: To solve model (1), the submodel corresponding to $f^{-}$would be solved firstly because of the following reasons:

(1) From Lemma 1, the solutions of the submodel corresponding to $f^{-}\left[\right.$i.e. $x_{\text {jopt }}^{-}(j=1,2, \ldots, k)$ and $x_{\text {jopt }}^{+} \quad(j=k+1$, $k+2, \ldots, n)]$ are identified from set $Q^{-}$. Since $Q^{-} \subseteq Q^{+}, \exists x_{j}^{ \pm}$ $\in Q^{+}$, such that $x_{j}^{+} \geq x_{j o p t}^{-}(j=1,2, \ldots, k)$ and $x_{j}^{-} \leq x_{j o p t}^{+} \quad(j$ $=k+1, k+2, \ldots, n)$.

(2) For model (1), the submodel corresponding to $f^{-}$would use relatively strict constraints (i.e. lower-bound values for the constraints' right-hand sides), and the submodel corresponding to $f^{+}$would use relatively relaxed constraints (i.e. upper-bound values for the right-hand sides). From theorem 2, additional constraints based on the solutions corresponding to $f^{-}$should be interposed for the submodel corresponding to $f^{+}$. This will help avoid violation of the best-case constraints as the decision variables fluctuated within the generated decision space.

Based on the above analysis, a robust two-step method (RTSM) will be developed to solve model (1). In this method, a conservative submodel (i.e. the submodel corresponding to $f^{-}$ when the objective is to be maximized) will be solved firstly; then an optimistic submodel (i.e. the submodel corresponding to $f^{+}$) will be derived based on solutions from the conservative submodel. In detail, the conservative submodel can be presented as follows (assume $b_{i}^{ \pm}>0, f^{ \pm}>0$ ):

$\operatorname{Max} f^{-}=\sum_{j=1}^{k} c_{j}^{-} x_{j}^{-}+\sum_{j=k+1}^{n} c_{j}^{-} x_{j}^{+}$,

subject to:

$\sum_{j=1}^{k}\left|a_{i j}^{ \pm}\right|^{+} \operatorname{Sign}\left(a_{i j}^{ \pm}\right) x_{j}^{-}+\sum_{j=k+1}^{n}\left|a_{i j}^{ \pm}\right|^{-} \operatorname{Sign}\left(a_{i j}^{ \pm}\right) x_{j}^{+} \leq b_{i}^{-}, i=1,2, \ldots, m$

$x_{j}^{-} \geq 0, j=1,2, \ldots, k$

$x_{j}^{+} \geq 0, j=k+1, k+2, \ldots, n$

From submodel (14), solutions of $x_{\text {jopt }}^{-}(j=1,2, \ldots, k)$ and $x_{\text {jopt }}^{+}(j=k+1, k+2, \ldots, n)$ can be obtained. These solutions will be incorporated into the optimistic submodel. The third criterion (as shown in Theorem 2) will then be employed to derive additional constraints that can help prevent from violation of the best-case constraints. Thus, the optimistic submodel can be formulated as follows (assume $b_{i}^{ \pm}>0, f^{ \pm}>0$ ):

$\operatorname{Max} f^{+}=\sum_{j=1}^{k} c_{j}^{+} x_{j}^{+}+\sum_{j=k+1}^{n} c_{j}^{+} x_{j}^{-}$

subject to:

$\sum_{j=1}^{k}\left|a_{i j}^{ \pm}\right|^{-} \operatorname{Sign}\left(a_{i j}^{ \pm}\right) x_{j}^{+}+\sum_{j=k+1}^{n}\left|a_{i j}^{ \pm}\right|^{+} \operatorname{Sign}\left(a_{i j}^{ \pm}\right) x_{j}^{-} \leq b_{i}^{+}, i=1,2, \ldots, m$.

$\sum_{j=1}^{l_{i 1}} a_{i j}^{-} x_{j}^{+}+\sum_{j=l_{i 1}+1}^{k} a_{i j}^{-} x_{j o p t}^{-}+\sum_{j=k+1}^{l_{i 2}} a_{i j}^{-} x_{j}^{-}+\sum_{j=l_{i 2}+1}^{n} a_{i j}^{-} x_{j o p t}^{+} \leq b_{i}^{+}$ 
$x_{j}^{+} \geq x_{j o p t}^{-} \quad j=1,2, \ldots, k$

$x_{j}^{-} \leq x_{j o p t}^{+} \quad j=k+1, k+2, \ldots, n$

$x_{j}^{+} \geq 0, j=1,2, \ldots, k$

$x_{j}^{-} \geq 0, j=k+1, k+2, \ldots, n$

where $c_{j}^{ \pm} \geq 0(j=1,2, \ldots, k)$, and $c_{j}^{ \pm} \leq 0(j=k+1, k+$ $2, \ldots, n) ; \quad a_{i j}^{ \pm} \geq 0\left(j=1,2, \ldots, l_{i 1} ; j=l_{i 2}+1, l_{i 2}+2, \ldots, n\right)$ and $a_{i j}^{ \pm} \leq 0\left(j=l_{i 1}+1, l_{i 1}+2, \ldots, l_{i 2}\right)$, where $l_{i 1} \leq k$, and $l_{i 2} \geq k$.

Hence, solutions of $x_{\text {jopt }}^{+}(j=1,2, \ldots, k)$ and $x_{\text {jopt }}^{-}(j=k+$ $1, k+2, \ldots, n)$ can be generated through submodel (15). Therefore, the ultimate solutions for model (1) are: $x_{j o p t}^{ \pm}=$ $\left[x_{\text {jopt }}^{-}, x_{\text {jopt }}^{+}\right]$and $f_{\text {opt }}^{ \pm}=\left[f_{\text {opt }}^{-}, f_{\text {opt }}^{+}\right]$.

\section{Illustrative Case Study of Environmental Management}

An illustrative case of interval parameter linear programming presented by Zhou et al. (2008) would be analyzed to show the solution process of RTSM:

$\operatorname{Max} f^{ \pm}=[26,30] x_{1}^{ \pm}-[5.5,6.0] x_{2}^{ \pm}$

subject to:

$[8,10] x_{1}^{ \pm}-[12,14] x_{2}^{ \pm} \leq[3.8,4.2]$

$[1.0,1.1] x_{1}^{ \pm}+[0.19,0.2] x_{2}^{ \pm} \leq[6.5,7]$

$x_{1}^{ \pm} \geq 0, \quad x_{2}^{ \pm} \geq 0$

In the above problem, it is assumed that the objective is to maximize economic return, while constraints (16b) and (16c) denote respectively limitations in resource availability and pollutant emission allowance.

Figure 1 shows a graphical presentation of the decision space for model (16). Based on constraints (16b) and (16c), the decision space is firstly divided into eight zones (i.e. Zones A, B, C, D, E, F, G, H, and I). Different zones would satisfy or violate different constraints. For example, any point in zone $\mathrm{C}$ is infeasible for constraint (16b); in contrast, all solutions in zone $\mathrm{G}$ satisfy constraint (16b) regardless the variation of its uncertain coefficients. In detail, zones B, C, D, E, and F are infeasible for model (16) since they would lead to absolute infeasibility. This means that the points located in those zones would violate at least one of the two best-case constraints for model (16), which are presented as the dash lines in Figure 1. Conversely, the solutions in zone $\mathrm{H}$ are absolutely feasible for model (16) since they satisfy the worst-case constraints; however, these solutions would lead to serious waste of resources since uncertainties of the right-hand-side coefficients are not consi-
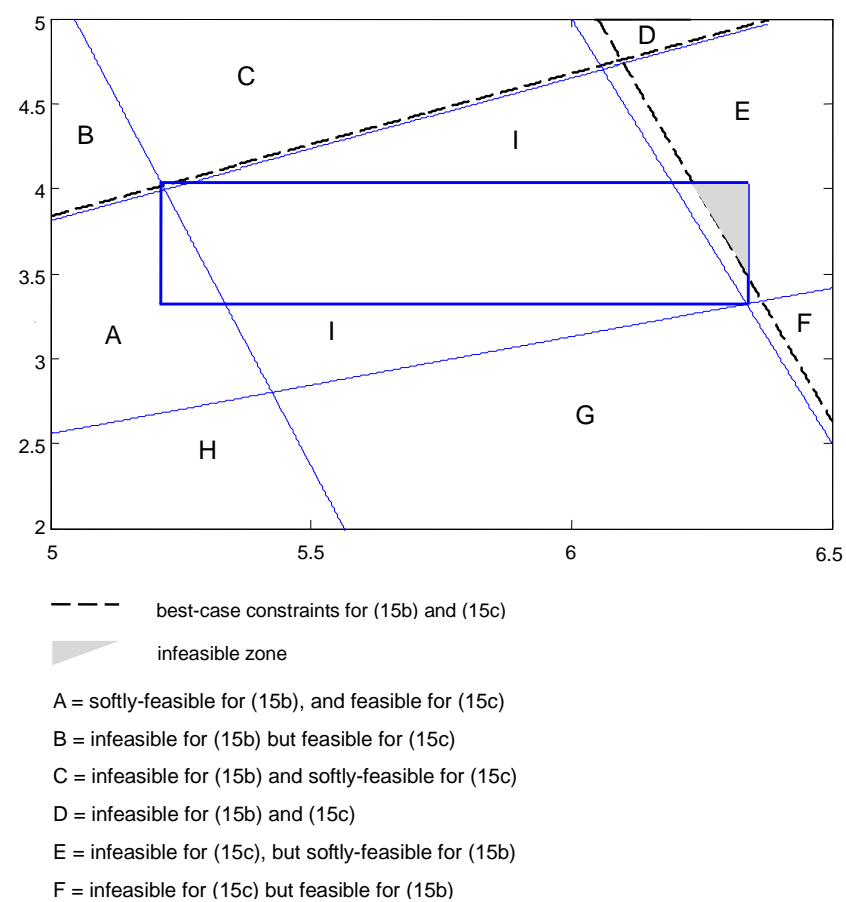

Figure 1. Inexact constraints and solution space obtained through TSM.

dered. Therefore, zones A, I and G would be potentially feasible, which were denoted as 'softly feasible zones" by Huang et al. (2002). In fact, the 'soft' concept corresponds to the existence of uncertainties, demonstrating the existence of a tradeoff between system benefit and constraint-violation risk (Huang et al., 2002). A high benefit corresponds to a high risk, and it is impossible to accomplish both high benefit and low risk simultaneously.

Based on the two-step method (TSM) of Huang et al. (1992), model (16) can be solved through formulating the following two submodels:

The first submodel:

$\operatorname{Max} f^{+}=30 x_{1}^{+}-5.5 x_{2}^{-}$

subject to:

$8 x_{1}^{+}-14 x_{2}^{-} \leq 4.2$

$1 x_{1}^{+}+0.2 x_{2}^{-} \leq 7$

$x_{1}^{+} \geq 0, \quad x_{2}^{-} \geq 0$

The second submodel:

$\operatorname{Max} f^{-}=26 x_{1}^{-}-6.0 x_{2}^{+}$

subject to:

$10 x_{1}^{-}-12 x_{2}^{+} \leq 3.8$ 


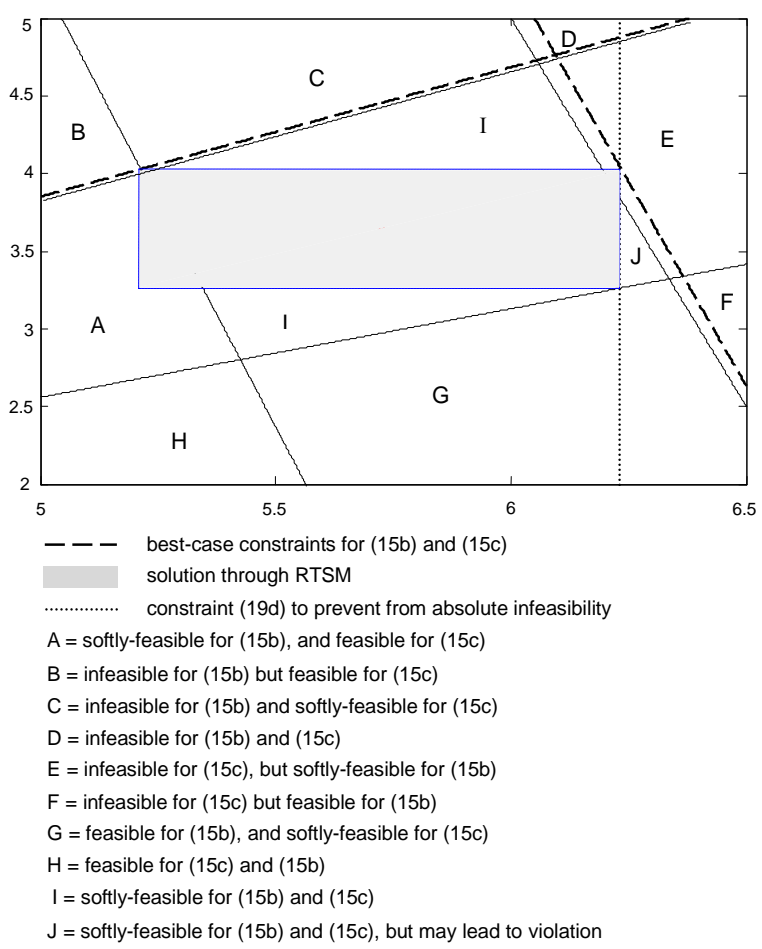

Figure 2. Inexact constraints and solution space obtained through RTSM.

$1.1 x_{1}^{-}+0.19 x_{2}^{+} \leq 6.5$

$x_{1}^{-} \leq x_{1 o p t}^{+}, \quad x_{2}^{+} \geq x_{2 o p t}^{-}$

$x_{1}^{-} \geq 0, \quad x_{2}^{+} \geq 0$

Thus, the solutions of model (16) through TSM are: $x_{1 \text { opt }}^{ \pm}=[5.21,6.34], x_{2 o p t}^{ \pm}=[3.32,4.03]$, and $f_{\text {opt }}^{ \pm}=[111.4$, 171.8].

The rectangle in Figure 1 shows the solution space obtained through TSM. It is obvious that this space contains a zone where the best-case constraints are violated when decision variables fluctuate within the obtained interval solutions. For example, for the best case of constraint (16c), if $x_{1}=x_{1 \text { opt }}^{+}=6.34, x_{2}$ $=x_{2 o p t}^{+}=4.03$, then we have $1 \times x_{1}+0.19 \times x_{2}=6.34+0.19$ $\times 4.03=7.11>7$. The shadowed zone in Figure 1 presents the solutions that would violate at least one best-case constraint.

Based on RTSM, model (16) can be transformed into two submodels. The first submodel can be presented as follows:

$\operatorname{Max} f^{-}=26 x_{1}^{-}-6.0 x_{2}^{+}$

subject to:

$10 x_{1}^{-}-12 x_{2}^{+} \leq 3.8$

$1.1 x_{1}^{-}+0.19 x_{2}^{+} \leq 6.5$ $x_{1}^{-} \geq 0, \quad x_{2}^{+} \geq 0$

The solutions are $x_{1 o p t}^{-}=5.21, x_{2 o p t}^{+}=4.03$ and $f_{\text {opt }}^{-}=$ 111.38. Thus, the second submodel can be expressed as:

$\operatorname{Max} f^{+}=30 x_{1}^{+}-5.5 x_{2}^{-}$

subject to:

$8 x_{1}^{+}-14 x_{2}^{-} \leq 4.2$

$1 x_{1}^{+}+0.2 x_{2}^{-} \leq 7$

$1 x_{1}^{+}+0.19 x_{2 o p t}^{+} \leq 7$

$x_{1}^{+} \geq x_{1 o p t}^{-}, \quad x_{2}^{-} \leq x_{2 o p t}^{+}$

$x_{1}^{+} \geq 0, \quad x_{2}^{-} \geq 0$

The solutions of submodel (20) are $x_{1 \text { opt }}^{+}=6.23, x_{2 o p t}^{-}=$ 3.26 and $f_{\text {opt }}^{+}=169.1$. Consequently, the ultimate solutions of model (16) are: $x_{1 o p t}^{ \pm}=\left[\begin{array}{lll}5.21,6.23\end{array}\right], x_{2 o p t}^{ \pm}=[3.26,4.03]$, and $f_{\text {opt }}^{ \pm}=[111.38,169.1]$.

With the above RTSM, an additional constraint [i.e. constraint (20d) presented as dotted line in Figure 2] is proposed to prevent from violation of the best-case constraint. Through this constraint [i.e. constraint (20d)], zone $\mathrm{J}$ is excluded from the solution space. The shadowed zone in Figure 2 shows the ultimate solution space obtained through RTSM. It is indicated that the generated solutions are at least softly feasible in reference to the given constraints (i.e. no absolute violation for constraints).

Model (16) can also be solved through approaches of MILP (Zhou et al., 2008), ThSM-I and ThSM-II (Cao and Huang, 2011). The solutions are presented in Table 1. As shown in Figure 3, the rectangular with solid lines is the solution space obtained through MILP. MILP can ensure no violation of the bestcase constraints (present as dash lines in Figure 3). However, it results in a significantly shrunk solution space, leading to serious loss of many feasible decision alternatives. Figures 4 and 5 show the solutions obtained through ThSM-I and ThSM-II. They would not violate the best-case constraints of model (16). However, compared with RTSM, the two approaches would compress the solution space from all dimensions, which may lead to relatively more loss of feasible decision alternatives; it also requires feasibility test which may be redundant for largescale cases. Particularly when the study cases involve many variables and constraints, the feasibility test may lead to the issue of great computational requirement. In comparison, the solution space acquired through RTSM covers a relatively larger space of the "softly feasible" zone, and can thus avoid significant loss of decision-related information. Also, the procedures of RTSM are not complicated and will not lead to great computational requirement. 
Table 1. Solutions for Model (16) through the Previous Solution Methods

\begin{tabular}{lllll}
\hline & $\begin{array}{l}\text { TSM } \\
\text { (Huang et al., 1992) }\end{array}$ & $\begin{array}{l}\text { MILP } \\
\text { (Zhou et al., 2008) }\end{array}$ & $\begin{array}{l}\text { ThSM-I } \\
\text { (Cao and Huang, 2011) }\end{array}$ & $\begin{array}{l}\text { ThSM-II } \\
\text { (Cao and Huang, 2011) }\end{array}$ \\
\cline { 3 - 5 } & & $\mathrm{q}=0.833$ & $\mathrm{q}_{1}=0.813, \mathrm{q}_{2}=1$ \\
\hline$x_{\text {lopt }}^{ \pm}$ & {$[5.21,6.34]$} & {$[5.30,6.25]$} & {$[5.32,6.23]$} \\
$x_{2 \text { opt }}^{ \pm}$ & {$[3.32,4.03]$} & {$[3.37,6.34]$} & {$[3.38,3.97]$} & {$[3.32,4.03]$} \\
$f_{\text {opt }}^{ \pm}$ & {$[111.4,171.8]$} & {$[98.0,171.8]$} & {$[114.1,168.78]$} & {$[114.0,168.77]$} \\
\hline
\end{tabular}

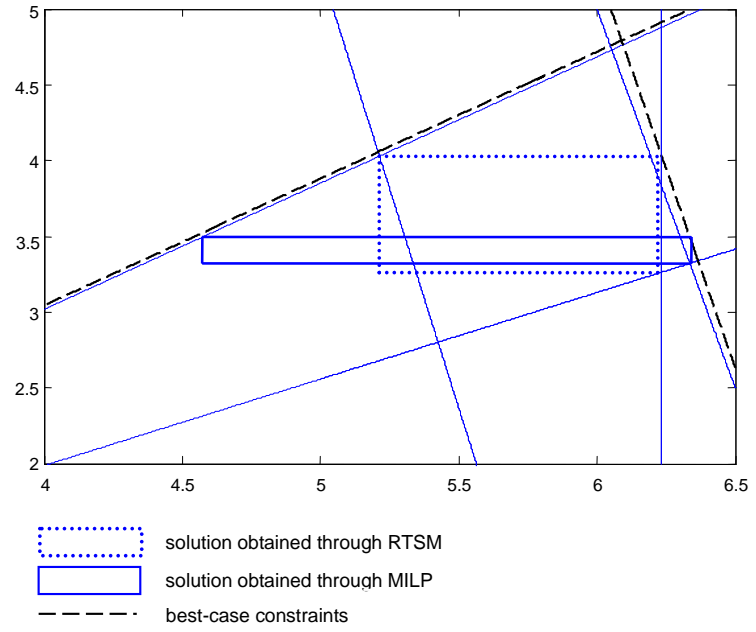

Figure 3. Solutions obtained through MILP and RTSM.

\section{Conclusions}

(1) A robust two-step method (RTSM) has been advanced to solve interval linear programming (ILP) problems. It improves upon the two-step method (TSM) proposed by Huang et al. (1992) through incorporating additional constraints into solution procedures to avoid absolute violation. Compared with TSM, RTSM can guarantee no violation of the best-case constraints when decision variables fluctuated within their solution spaces. RTSM has been applied to a simple case related to environmental management. The results demonstrate applicability of the developed methodology.

(2) Comparisons between the RTSM and the previous solution methods have been undertaken. Different solution spaces have been obtained. It is indicated that: (a) RTSM would generate a relatively larger solution space than other ILP solution methods, and can thus avoid significant loss of decisionrelated information. (b) In comparison with the three-step method (ThSM), the solution process of RTSM is much simpler. RTSM would not require feasibility test and model constriction. This would be meaningful for its practical applications, since the feasibility test may lead to high computational requirements for problems with a large number of constraints.

(3) Although this study is a new attempt to improve upon the two-step method as proposed by Huang et al. (1992), it is desired that more real-world case studies be undertaken to demonstrate its practical applicability.

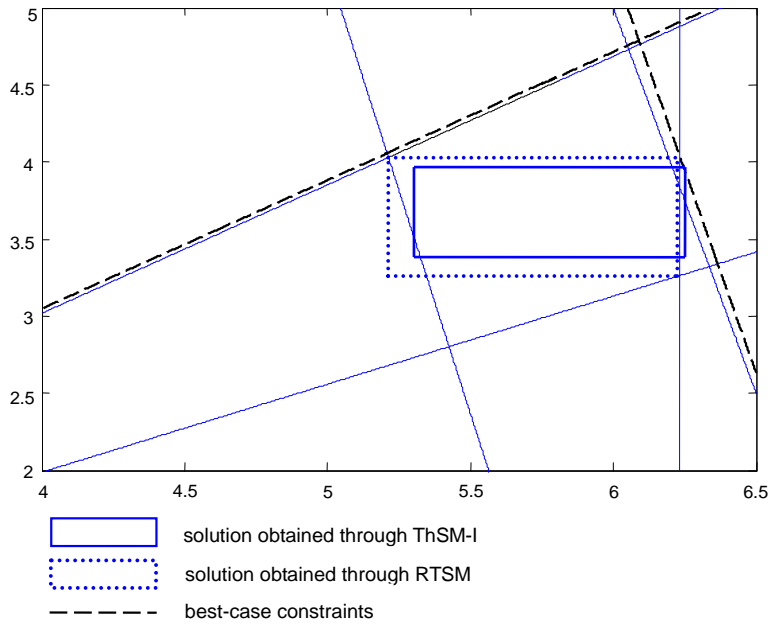

Figure 4. Solutions obtained ThSM-I and RTSM.

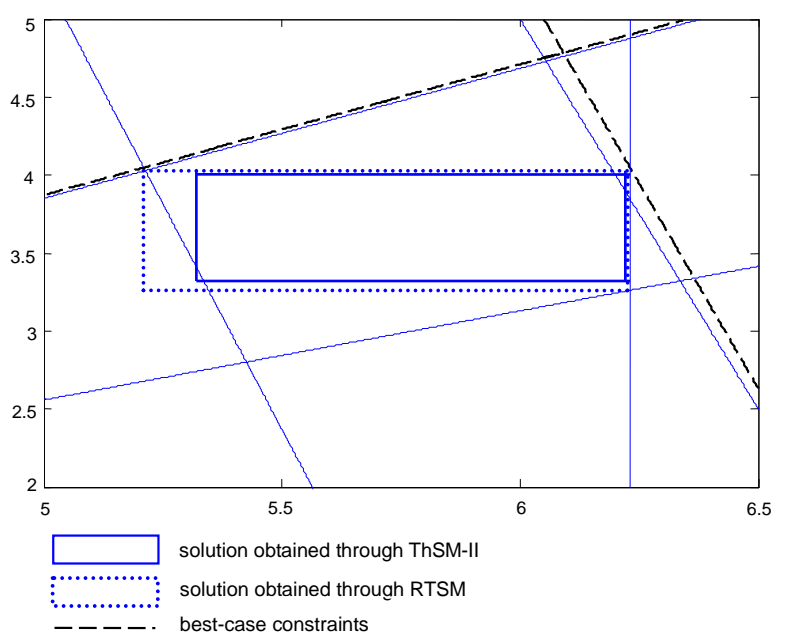

Figure 5. Solutions obtained through ThSM-II and RTSM.

Acknowledgments. This research was supported by the Program for Innovative Research Team (IRT1127), the MOE Key Project Program (311013), the Natural Science and Engineering Research Council of Canada, and the Major Project Program of the Natural Sciences Foundation (51190095). The authors are grateful to the editor and the anonymous reviewers for their insightful comments and suggestions. 


\section{References}

Bloemhof-Ruwaard, J.M., Van Beek, P., Hordijk, L., and Van Wassenhove, L.N. (1995). Interactions between operational research and environmental management. Eur. J. Oper. Res., 85(2), 22.9243. http://dx.doi.org/10.1016/0377-2217(94)00294-M

Chang, N.B., and Lu, H.Y. (1997). A new approach for long-term planning of solid waste management system using fuzzy global criterion. J. Environ. Sci. Health. (Part A), 32(4), 1025-1047.

Chang, N.B. and Wang, S.F. (1997). A fuzzy goal programming approach for the optimal planning of metropolitan solid waste management systems. Eur. J. Oper. Res., 99(2), 303-321. http://dx. doi.org/10.1016/S0377-2217(96)00024-0

Chinneck, J.W. and Ramada, K. (2000). Linear programming with interval coefficients. J. Oper. Res. Soc., 51(2), 209-220.

Fan, Y.R., Huang, G.H., Li, Y.P., Cao, M.F. and Cheng, G.H. (2009). A Fuzzy Linear Programming Approach for Municipal Solid-Waste Management under Uncertainty. Eng. Optimiz., 41(12), 1081-1101. http://dx.doi.org/10.1080/03052150902866569

Fan, Y.R., Huang, G.H., Guo, P., and Yang, A.L. (2012). Inexact twostage stochastic partial programming: application to water resources management under uncertainty. Stoch. Env. Res. Risk. A., 26(2), 281-293. http://dx.doi.org/10.1007/s00477-011-0504-6

Fan, Y.R., Huang, G.H., and Veawab, A. (2012). A Generalized Fuzzy Linear Programming Approach for Environmental Management Problem under Uncertainty. J. Air Waste Manage., 62(1), 72-86. http://dx.doi.org/10.1080/10473289.2011.628901

Fiedler, M., Nedoma, J., Ramik, J., Rohn, J., and Zimmermann, K. (2006). Linear optimization problems with inexact data, Springer, New York.

He, L., Huang, G.H., Zeng, G.M., and Lu, H.W. (2008). Identifying optimal regional solid waste management strategies through an inexact integer programming model containing infinite objectives and constraints. Waste Manage., 29(1), 21-31. http://dx.doi.org/10. 1016 /j.wasman.2008.02.003

Huang, G.H., Baetz, B.W., and Patry, G.G. (1992). A grey linear programming approach for municipal solid waste management planning under uncertainty. Civil Eng. Syst., 9(4), 319-335. http://dx. doi.org/10.1080/02630259208970657

Huang, G.H., Baetz, B.W., and Patry, G.G. (1995). Grey fuzzy integer programming: An application to regional waste management planning under uncertainty. Socio Econ. Plan Sci., 29(1), 17-38. http:// dx.doi.org/10.1016/0038-0121(95)98604-T

Huang, G.H., and Cao, M.F., (2011). Analysis of Solution Methods for Interval Linear Programming. J. Environ. Inform., 17(2): 54-64. http://dx.doi.org/10.3808/jei.201100187

Huang, G.H., and Chang, N.B. (2003). The Perspectives of Environmental Informatics and Systems Analysis. J. Environ. Inform., 1(1), 1-6. http://dx.doi.org/10.3808/jei.200300001

Huang, G.H. (1998). A hybrid inexact-stochastic water management model. Eur. J. Oper. Res., 107(1), 137-158. http://dx.doi.org/10.10 16/S0377-2217(97)00144-6

Huang, Y.F., Baetz, B.W., Huang, G.H., and Liu, L. (2002). Violation analysis for solid waste management systems: An interval fuzzy programming approach. J. Environ. Manage., 65(4), 431-446. http://dx.doi.org/10.1006/jema.2002.0566

Karmakar, S. and Mujumdar, P.P. (2006a). Grey fuzzy optimization model for water quality management of a river system. Adv. Water Resour., 29(7), 1088-1105. http://dx.doi.org/10.1016/j.advwatres.2 006.04.003

Karmakar, S. and Mujumdar, P.P. (2006b). An inexact optimization approach for river water quality management. J. Environ. Manage., 81(3), 233-248. http://dx.doi.org/10.1016/j.jenvman.2005.10.009
Li, Y.P., Huang, G.H., and Nie, S.L. (2006). An interval-parameter multi-stage stochastic programming model for water resources management under uncertainty. Adv. Water Resour, 29(5), 776789. http://dx.doi.org/10.1016/j.advwatres.2005.07.008

Li, Y.P., Huang, G.H., Nie, S.L., and Qin, X.S. (2007). ITCLP: An inexact two-stage chance-constrained program for planning waste management systems. Resour. Conserv. Recy., 49(3), 284-307. http: //dx.doi.org/10.1016/j.resconrec.2006.03.017

Li, Y.P., Huang, G.H., Nie, X.H., and Nie, S.L. (2008). A two-stage fuzzy robust integer programming approach for capacity planning of environmental management systems. Eur. J. Oper. Res., 189(2), 399-420. http://dx.doi.org/10.1016/j.ejor.2007.05.014

Liu, Y., Guo, H.C., Zhou, F., Qin, X.S., Huang, K., and Yu, Y.J. (2008). Inexact Chance-Constrained Linear Programming Model for Optimal Water Pollution Management at the Watershed Scale. $J$. Water Res. Pl. - ASCE, 134(4), 347-356. http://dx.doi.org/10.1061/ (ASCE)0733-9496(2008)134:4(347)

Lv, Y., Huang, G.H., Li, Y.P., Yang, Z.F., Liu, Y., and Cheng, G.H. (2010). Planning Reginal Water Resources System Using an Interval Fuzzy Bi-liver Programming Method. J. Environ. Inform., 16(2), 43-56. http://dx.doi.org/10.3808/jei.201000177

Nie, X.H., Huang, G.H., Li, Y.P., and Liu, L. (2007). IFRP: A hybrid interval-parameter fuzzy robust programming approach for waste management planning under uncertainty. J. Environ. Manage., 84 (1), 1-11. http://dx.doi.org/10.1016/j.jenvman.2006.04.006

Oliveira, C., and Antunes, C.H. (2007). Multiple objective linear programming models with interval coefficients - an illustrated overview. Eur. J. Oper. Res., 181(3), 1434-1463. http://dx.doi.org/10. 1016/j.ejor.2005.12.042

Ozdemir, M.S., and Saaty, T.L. (2006). The unknown in decision making: What to do about it. Eur. J. Oper. Res., 174(1), 349-359. http://dx.doi.org/10.1016/j.ejor.2004.12.017

Ruszcyński, A. (1997). Decomposition methods in stochastic programming. Math. Program., 79(1-3), 333-353. http://dx.doi.org/10. 1007/BF02614323

Sengupta, A., Pal, T.K., and Chakraborty, D. (2001). Interpretation of inequality constraints involving interval coefficients and a solution to interval linear programming. Fuzzy Set. Syst., 119(1), 129-138. http://dx.doi.org/10.1016/S0165-0114(98)00407-2

Sun, W., and Huang, G.H. (2010). Inexact Piecewise Quadratic Programming for Waste Flow Allocation under Uncertainty and Nonlinearity. J. Environ. Inform., 16(2), 80-93. http://dx.doi.org/10.380 $8 /$ jei.201000180

Tong, S.C. (1994). Interval number, fuzzy number linear programming. Fuzzy Set. Syst., 66(3), 301-306. http://dx.doi.org/10.1016 /0165-0114(94)90097-3

van Beek, P., Fortuin, L., and Van Wassenhove, L.N. (1992). Operational Research and the Environment. Environmetal \& Resource Econo., 2(6), 635-639. http://dx.doi.org/10.1007/BF00330288

Yan, X.P., Ma, X.F., Huang, G.H., and Wu, C.Z. (2010). An Inexact Transportation Planning Model for Supporting Vehicle Emissions Management. J. Environ. Inform., 15(2), 87-98. http://dx.doi.org/ $10.3808 /$ jei.201000169

Young, R.A. (2001). Uncertainty and the Environment: Implications for Decision Making and Environmental Policy, Edward Elgar, Cheltenham, UK.

Zhou, F., Guo, H.C., Huang, K., and Huang, G.H. (2008). The Interval Linear Programming: A Revisit. J. Environ. Inform., 11(1), 1-10. http://dx.doi.org/10.3808/jei.200800105

Zou, R., Liu, Y., Liu, L., and Guo, H.C. (2010). REILP Approach for Uncertainty-Based Decision Making in Civil Engineering. $J$. Comput. Civil Eng. -ASCE, 24(4), 357-364. http://dx.doi.org/10. 1061/(ASCE)CP.1943-5487.0000037 